TRANSACTIONS OF THE

AMERICAN MATHEMATICAL SOCIETY

Volume 173, November 1972

\title{
A RADON-NIKODÝM THEOREM FOR OPERATOR-VALUED MEASURES
}

BY

\author{
HUGH B. MAYNARD
}

\begin{abstract}
The purpose of this paper is to obtain a characterization of indefinite integrals of vector-valued functions with respect to countably additive operator-valued measures with finite variation. This result is then specialized to several simpler situations.
\end{abstract}

0 . Introduction. The setting considered in this paper is that of a measurable space $(X, \mathfrak{S})$, where $X \in \mathfrak{d}$ for simplicity, together with a vector measure $\mu: \mathfrak{S}$ $\rightarrow L\left[B, B_{1}\right]$ with total finite variation $|\mu|$, where $B$ and $B_{1}$ are Banach spaces. $A$ "natural" space of integrable functions into $B$ is introduced which agrees with the space $L_{B}^{1}(\mu)$ defined by Dinculeanu $[1, \mathrm{p} .120]$, whenever $B=\mathrm{C}$ or $\mu$ is a scalar measure.

A general decomposition theorem for $(X, \mathcal{S}, \mu)$ is utilized to obtain a characterization of those $B_{1}$-valued measures which admit a representation as the integral of an integrable $B$-valued function with respect to $\mu$. This characterization is then used to obtain a theorem of Rieffel's [4, p. 466] in the setting of the Bochner integral. Several additional results, all apparently new, are obtained in the cases where either $B=C$ or $B=C^{n}$.

1. Preliminaries. In this section we shall develop a space of integrable functions which is naturally suited for integration. Dinculeanu $[1, \mathrm{p}, 120]$ introduces the. space of integrable functions $L_{B}^{1}(X, \mu)$ which has the advantage of being complete but unfortunately integration processes do not distinguish functions. ( $\left.{ }^{1}\right)$ We plan to remedy this at the expense of completeness.

First we shall establish a useful decomposition result.

Definition 1.1. A set property $P$ is said to be local in $(X, \mathcal{S}, \mu)$ if, for each $E \in \mathcal{S}^{+}=\{E \in \mathcal{S}|| \mu \mid(E)>0\}$, there exists $F \in \mathcal{S}^{\dagger}, F \subset E$, such that $F$ has property $P$.

Definition 1.2. A set property $P$ is a null difference property in $(X, \mathcal{S}, \mu)$ if whenever $F \in \mathcal{S}^{+}$has property $P$ and $E \in \mathcal{S},|\mu|(E)=0$, then both $F \cup E$ and $F \sim E$ have property $P$.

Received by the editors August 2, 1971.

AMS (MOS) subject classifications (1970). Primary 46G10, 46E40, 46G05. integral.

Key words and phrases. Radon-Nikodým theorem, operator-valued measures, Bochner

(1) There may exist distinct equivalence classes whose integral values are all equal. 
A simple exhaustion argument quickly establishes the following decomposition result.

Lemma 1.1. If $P$ is a local null difference property in $(X, \mathcal{S}, \mu)$, then there exists a pairwise disjoint set $\left\{E_{i}\right\}_{i \in I} \subset \mathcal{S}^{+}, I$ either finite or countable, such that

(i) $X=\bigcup_{i \in I} E_{i}$ and,

(ii) each $E_{i}$, $i \in I$, has property $P$.

Our space of integrable functions is constructed by considering those functions which are almost every where limits of Cauchy sequences of measurable stepfunctions and decomposing into equivalence classes modulo the null functions. The difference arises from the seminorm placed on the space of measurable stepfunctions; we use $\rho(f)=\left|\mu_{f}\right|(X)$, where if $f=\Sigma_{i=1}^{n} x_{i} \chi_{E_{i}}, x_{i} \in B, \mu_{f}(E)=\int_{E} f d \mu=$ $\sum_{i=1}^{n} \mu\left(E \cap E_{i}\right)\left(x_{i}\right)$, while Dinculeanu uses $\rho(f)=\int_{X}\|f\| d|\mu|$ in constructing $L_{B}^{1}(X, \mathcal{S}, \mu)$. Let $\mathfrak{Q}_{B}(X, \mu)$ be the resulting space of equivalence classes of integrable functions with norm $\|\cdot\|_{\mathcal{P}}$. The integral is defined by the normal limiting process. We shall now proceed to identify functions with their equivalence classes, regardless of the space they exist in.

Using standard arguments we can establish the following lemmas.

Lemma 1.2. If $f \in \mathscr{L}_{B}(X, \mu)$, then $\|/\|_{\mathfrak{L}}=\left|\mu_{f}\right|(X)$.

Thus two functions are in the same equival ence class iff their integrals coincide.

Lemma 1.3. If $f \in \mathscr{L}_{B}(X, \mu)$ and $f \in L_{B}^{1}(X, \mu)$ then

$$
\|f\|_{\mathfrak{S}} \leq \int_{X}\|f\| d|\mu|=\|f\|_{1} \text {. }
$$

Theorem 1.1. If $\mu$ is $\mathbf{C}$-valued or if $B=\mathbf{C}$, then

$$
\varrho_{B}(X, \mu)=L_{B}^{1}(X, \mu) \text {. }
$$

This follows from the identity $\left|\mu_{f}\right|(X)=\int_{X}\|f\| d|\mu|$ for all measurable stepfunctions $f$.

Two additional lemmas will also be necessary.

Lemma 1.4. If $f \in \mathfrak{L}_{B}(X, \mu)$, then $\mu_{f}$ is a countably additive $B_{1}$-valued measure.

Lemma 1.5. If $f \in \mathfrak{L}_{B}(X, \mu)$, then given any $E \in \mathcal{S}^{+}$, there exists $F \subset E, F \in$ $\mathcal{S}^{+}$, such that the essential range of $f$ on $F, \operatorname{er}_{F}(f)$, is compact.

2. A Radon-Nikodým theorem. We shall continue using the setting developed in $\$ 1$ throughout this section. In addition we will assume we have another vectorvalued measure $m: \mathcal{S} \rightarrow B_{1}$ such that $m$ has finite variation.

Definition 2.1. $m$ is said to be absolutely continuous with respect to $\mu$ 
$(m \ll \mu)$ iff $|\mu|(E)=0, E \in \mathcal{S}$, implies $|m|(E)=0$.

In the remainder of this paper $m$ will be considered to be $\mu$-continuous.

Definition 2.2. If $\epsilon>0$ and $E \epsilon \mathcal{S}^{+}$, let

$$
A_{m}(E, \epsilon)=\{b \in B|\|m(F)-\mu(F)(b)\| \leq \epsilon| \mu \mid(F), \forall F \in \mathcal{S}, F \subset E\} .
$$

If the measure $m$ is obvious, we will neglect the subscript and write only $A(E, \epsilon)$.

Definition 2.3. A set $Y \in \mathcal{S}^{+}$is said to be semilocalized in a set $K \subset B$ with respect to $(\mu, m)$ if, for all $\epsilon>0$, there exists $F \subset Y, F \in \mathcal{S}^{+}$, such that $A_{m}(F, \epsilon) \cap K \neq \varnothing$.

A set $Y \in \mathcal{S}^{+}$is said to be localized in $K$ with respect to $(\mu, m)$ if, for each $E \subset Y, E \in \mathcal{S}^{+}, E$ is semilocalized in $K$.

If $\mu$ and $m$ are clear from context we shall merely say that $Y$ is localized in $K$.

The following lemmas follow easily from the definitions and exhaustion techniques.

Lemma 2.1. If $E$ is semilocalized in $K$ and $F \supset E, F \in \mathcal{S}$, then $F$ is semilocalized in $K$.

Lemma 2.2. If $Y$ is localized in $K=K_{1} \cup K_{2}$ and $E \subset Y, E \in \mathfrak{S}^{+}$, then either $E$ is semilocalized in $K_{1}$ or $E$ is semilocalized in $K_{2}$.

Lemma 2.3. If $Y$ is localized in $K=K_{1} \cup K_{2}$, then $Y=Y_{1} \cup Y_{2}$, where either $Y_{i}=\varnothing$ or $Y_{i}$ is localized in $K_{i}, i=1,2$, and $Y_{1} \cap Y_{2}=\varnothing$.

Corollary. If $Y$ is localized in $K=\bigcup_{i=1}^{n} K_{i}$, then $Y=\bigcup_{i=1}^{n} Y_{i}$, where $\left\{Y_{i}\right\}_{i=1}^{n}$ are pairwise disjoint and either $Y_{i}=\varnothing$ or $Y_{i}$ is localized in $K_{i}, 1 \leq$ $i \leq n$.

Lemma 2.4. If $Y$ is localized in $K$, then, for each $\epsilon>0$, there exists a dis. joint collection $\left\{E_{i}\right\}_{i \in I} \subset \mathcal{S}^{+}$, where $I$ is either finite or denumerable, such that $Y=\bigcup_{i \in I} E_{i}$ and $A\left(E_{i}, \epsilon\right) \cap K \neq \varnothing$.

Proof. This follows immediately from Lemma 1.1 since the set property $A(E, \epsilon) \cap K \neq \varnothing$ is a local null difference property.

Theorem 2.1. Let $(X, \mathcal{S})$ be a measurable space, $X \in \mathcal{S}$, and let $\mu: \mathcal{S} \rightarrow$ $L\left[B, B_{1}\right]$ be a measure with total finite variation where $B$ and $B_{1}$ are Banach spaces. Let $m: \mathcal{S} \rightarrow B_{1}$ be a measure on $\mathcal{S}$. Then $m$ is the indefinite integral with respect to $\mu$ of a function $f \in \mathcal{Q}_{B}(X, \mu)$ iff

(i) $m \ll \mu$,

(ii) $|m|(X)<\infty$, and

(iii) for each $E \in \mathcal{S}^{+}$, there exists $F \subset E, F \in \mathcal{S}^{+}$, and a compact set $K \subset B$ such that $F$ is localized in $K$. 
Before we prove this theorem we shall prove two preparatory lemmas.

Lemma 2.5. If $m$ satisfies conditions (i), (ii) and (iii) of Theorem 2.1, then there exists a disjoint set $\left\{X_{i}\right\}_{i \in I} \subset \mathcal{S}^{+}, I$ either finite or denumerable, such that $X=\bigcup_{i \in I} X_{i}$ and for each $i \in I$ there exists a compact set $K_{i} \subset B$ such that $X_{i}$ is localized in $K_{i}$.

Proof. This follows from Lemma 1.1 since conditions (i), (ii), (iii) imply that the existence of a compact set $K$, such that $F$ is localized in $K$, is a local null difference property.

Because of the complicated construction to follow we will need a somewhat elaborate indexing notation. To this end we will introduce the following notation.

If $z=\left(z_{1}, \ldots, z_{n}\right) \in \mathbf{N}^{n}$, we let $\pi(z)=\left(z_{1}, \ldots, z_{n-1}\right) \in \mathbf{N}^{n-1}$ and $(z, i)=$ $\left(z_{1}, \cdots, z_{n}, i\right) \in \mathbf{N}^{n+1}$, for $i \in \mathbf{N}$, suppressing for convenience the dependence upon $n$.

The following lemma corresponds roughly, in the Bochner integral case, to partitioning a set $Y$ into sets whose average range has a small diameter.

Lemma 2.6. If $m$ satisfies conditions (i), (ii) and (iii), of Theorem 2.1, and if $Y \in \mathcal{S}^{+}$is localized in a compact set $K \subset B$, there exists a sequence of measurable finite partitions $\left\{\pi_{n}\right\}_{n=1}^{\infty}$ of $Y$ such that

(i) $Y=\bigcup_{z \in A_{n}} Y_{z}^{n}$ where $\pi_{n}=\left\{Y_{z}^{n}\right\}_{z \in A_{n}}$, and the index set $A_{n}$ is a finite subset of $\mathbf{N}^{n}$;

(ii) each $Y_{z}^{n}$ is localized in a compact set $K_{z}^{n} \subset B$ such that the diameter $\left(K_{z}^{n}\right)<1 / 2^{n}$, and

(iii) $\pi_{n}$ is finer than $\pi_{n-1}$ and $K_{\pi(z)}^{n-1} \supset K_{z}^{n}$ for all $z \in A_{n}$.

Proof. We will construct $\pi_{n}$ from $\pi_{n-1}$ in the following manner, starting with $\pi_{0}=\{Y\}, Y_{0}^{0}=Y A_{0}=\{0\}$, and $K_{0}^{0}=K$.

Assume $\left\{Y_{z}^{n}\right\}_{z \in A_{n}},\left\{K_{z}^{n}\right\}_{z \in A_{n}}$ satisfy (i), (ii) and (iii). Then for each $z \in A_{n}$, there exists, by the compactness of $K_{z}^{n},\left\{K_{(z, i)}^{n+1}\right\}_{i=1}^{m} z$, such that $K_{z}^{n}=\bigcup_{i=1}^{m z} K_{\left(z_{0} i\right)}^{n+1}$ where $K_{\left(z_{0} i\right)}^{n+1}$ are compact and the diameter $\left(K_{\left(z_{0} i\right)}^{n+1}\right)<1 / 2^{n+1}$.

For each $z \in A_{n}$, we have by the corollary to Lemma 2.3, $\left\{Y_{\left(z_{0} i\right)}^{n+1}\right\}_{i=1}^{m} z$ such that $Y_{z}^{n}=\bigcup_{i=1}^{m z} Y_{(z, i)}^{n+1}$ and either $Y_{(z, i)}^{n+1}=\varnothing$ or $Y_{(z, i)}^{n+1}$ is localized in $K_{(z, i)}^{n+1}$ $1 \leq i \leq m_{z}$. We now eliminate those $K_{(z, i)}^{n+1}$, and $Y_{(z, i)}^{n+1}$ where $Y_{(z, i)}^{n+1}=\varnothing$. If, after renumbering there are $n_{z}$ nonempty $Y_{\left(z_{0}, i\right)}^{n+1}$, we have $Y_{z}^{n}=\bigcup_{i=1}^{n z} Y_{\left(z_{0} i\right)}^{n+1}$, and $Y_{\left(z_{0}, i\right)}^{n+1}$ is localized in $K_{(z, i)}^{n+1}, 1 \leq i \leq z_{z}$, and further $K_{z}^{n} \supset \bigcup_{i=1}^{z_{z}} K_{\left(z_{0} i\right)^{\circ}}^{n+1}$

Let $A_{n+1}=\left\{(z, i) \mid z \in A_{n}\right.$ and $\left.1 \leq i \leq n_{z}\right\}$. Thus letting $\pi_{n+1}=\left\{Y_{z}^{n+1}\right\}_{z \in A_{n+1}}$, it is clear that this procedure generates the desired sequence of partitions.

Proof of Theorem 2.1. ( $\Leftarrow)$ ). Assume $m$ satisfies conditions (i), (ii) and (iii). Then by Lemma 2.5. we have that $X=\bigcup_{p \in I} X_{p}$, where $\left\{X_{p}\right\}_{p \in I}$ is a disjoint subset of $\mathcal{S}^{+}$such that each $X_{p}$ is localized in a compact set $K_{p} \subset B$. 
For ease in notation we will assume that $I$ is infinite for if $I$ is finite then $X$ itself is localized in the compact set $\bigcup_{p \in I} K_{p}$, and the argument reduces to the following argument for $X_{p}$. We will now proceed to define the desired function piecewise on each $X_{p}$.

Consider the measure space $\left(X_{p}, \mathcal{S}_{p}, \mu\right)$ where $\mathcal{S}_{p}$ is the restriction of $\mathcal{S}_{\text {to }}$ $X_{p}$. Then by Lemma 2.6 there exists a sequence of partitions of $X_{p},\left\{\pi_{n}\right\}_{n=1}^{\infty}$ satisfying the three conditions of Lemma 2.6.

For each $n$, define a stepfunction $f_{n}$ in the following manner. Let $N_{n}$ be the cardinality of the set $A_{n}$ where $\pi_{n}=\left\{Y_{z}^{n}\right\}_{z \in A_{n}}$. Then by Lemma $2.4, Y_{z}^{n}=$ $\bigcup_{i \in I_{z}^{n}} E_{z i}^{n}$ such that

$$
A_{m}\left(E_{z i}^{n}, 1 / 2^{n}\right) \cap K_{z}^{n} \neq \not
$$

Choose $l_{n z}$ such that

$$
|\mu|\left(Y_{z}^{n} \sim \bigcup_{i=1}^{l} E_{z i}^{n}\right)<\frac{1}{N_{n} 2^{n}}
$$

For each $E_{z i}^{n}, \quad 1 \leq i \leq l_{n z^{\prime}} z \in A_{n}$, choose $x_{z i}^{n} \in A_{m}\left(E_{z i}^{n}, 1 / 2^{n}\right) \cap K_{z}^{n}$. We then define

$$
f_{n}=\sum_{z \in A} \sum_{i=1}^{l} x_{z i}^{n} \circ \chi_{E_{z i}^{n}} .
$$

Since much of the remainder of the proof is computational only the key steps will be demonstrated. The sequence $\left\{f_{n}\right\}_{n=1}^{\infty}$ converges a.e. and is Cauchy in $\|\cdot\|_{\mathcal{Q}}$ which is demonstrated in the next three claims.

Claim 1. $|\mu|\left(X_{p} \sim \bigcup_{m=1}^{\infty}\left[\bigcap_{k=m}^{\infty}\left(\bigcup_{z \in A_{k}} \bigcup_{i=1}^{l_{n} z} E_{z i}^{k}\right)\right]\right)=0$.

Claim 2. $\left\{f_{n}\right\}_{n=1}^{\infty}$ is Cauchy in $\|\cdot\|_{\rho}$.

Proof. Let $Z_{k}=\bigcup_{z \in A_{k}} \bigcup_{i=1}^{l_{n z}} E_{z i}^{k}$

Let $\epsilon>0$ be given. Since $|m|$ is $|\mu|$-continuous there exists $\delta>0$ such that, whenever $|\mu|(E)<\delta$, then $|m|(E)<\delta / 4$. Choose $N \geq 1$ such that

$$
1 / 2^{N}<\min \left\{\epsilon / 4|\mu|\left(X_{p}\right), \delta\right\}
$$

We first make the observation that

$$
|\mu|\left(X_{p} \sim Z_{n}\right)<1 / 2^{n}
$$

Suppose $m, n \geq N$. Then

$$
\begin{aligned}
\left\|f_{n}-f_{m}\right\|_{Q} \leq \sup \{ & \sum_{k=1}^{r}\left\|\int_{B_{k} \cap\left(Z_{n}-Z_{m}\right)} f_{n} d \mu\right\|+\sum_{k=1}^{r}\left\|\int_{B_{k} \cap\left[Z_{m}-Z_{n}\right]} f_{m} d \mu\right\| \\
& \left.+\sum_{k=1}^{r} \sum_{z \in A_{n} ; z^{\prime} \in A_{m}} \sum_{i=1}^{l_{n z}} \sum_{\alpha=1}^{l_{m z}^{\prime}}\left\|\int_{B_{k} \cap E_{z i}^{n} \cap E_{z^{\prime} \alpha}^{m}} f_{n}-f_{m} d \mu\right\|\right\} \\
= & \sup \left\{S_{1}+S_{2}+S_{3}\right\}
\end{aligned}
$$


where the supremum is over all finite disjoint measurable partitions $\left\{B_{k}\right\}_{k=1}^{r}$ of $X_{p}$

Using the fact that $f_{n}=x_{z i}^{n}$ on $E_{z i}^{n}$ and that $x_{z i}^{n} \in A_{m}\left(E_{z i}^{n}, 1 / 2^{n}\right)$ and similarly for $f_{m}$, we can show the following bounds for $S_{1}, S_{2}$ and $S_{3}$.

$$
\begin{aligned}
& S_{1} \leq\left(1 / 2^{n}\right)|\mu|\left(Z_{n} \sim Z_{m}\right)+|m|\left(Z_{n} \sim Z_{m}\right), \\
& S_{2} \leq\left(1 / 2^{m}\right)|\mu|\left(Z_{m} \sim Z_{n}\right)+|m|\left(Z_{m} \sim Z_{n}\right), \text { and } \\
& S_{3} \leq\left(1 / 2^{n}\right)|\mu|\left(Z_{n} \cap Z_{m}\right)+\left(1 / 2^{m}\right)|\mu|\left(Z_{n} \cap Z_{m}\right) .
\end{aligned}
$$

Combining these bounds with the definition of $\delta$ and $N$ we see that

$$
\left\|f_{n}-f_{m}\right\|_{\mathcal{L}} \leq \epsilon
$$

and hence that $\left\{f_{n}\right\}_{n=1}^{\infty}$ is Cauchy in $\|\cdot\|_{\mathscr{Q}}$.

Claim 3. $f_{n}$ converges a.e. to a function $f^{p}$.

Proof. $\left\{f_{n}(t)\right\}_{n=1}^{\infty}$ converges for all $t \in \bigcup_{m=1}^{\infty} \bigcap_{k=m}^{\infty} Z_{k}$ since if $t \epsilon$ $\bigcap_{k=N}^{\infty} Z_{k}$ and $n \geq m>N$, then $f_{n}(t)$ and $f_{m}(t)$ are both in the same compact set $K_{z}^{m}$ with diameter $1 / 2^{m}$. Thus $f^{p} \in \mathscr{L}_{B}\left(X_{p}, \mu\right)$, and $f_{n} \rightarrow f^{p}$.

Claim 4. If $E \in \mathcal{S}_{p}$, then $m(E)=\int_{E} f^{p} d \mu$.

Proof. Let $\epsilon>0$ be arbitrary. Then choose $\delta>0$ such that $|\mu|(F)<\delta$ implies that $|m|(F)<\epsilon / 3$. Choose $n$ sufficiently large so that

(i) $\left\|f^{p}-f_{n}\right\|_{P}<\epsilon / 3$,

(ii) $1 / 2^{n}<\delta$, and

(iii) $\left(1 / 2^{n}\right)|\mu|\left(X_{p}\right)<\epsilon / 3$.

Now by adding and subtracting $f_{n}$ and using the fact that $x_{z i}^{n} \in A_{m}\left(E_{z i}^{n}, 1 / 2^{n}\right)$ we have

$$
\begin{array}{rl}
\| m(E)-\int_{E} & f d \mu \| \\
& \leq|m|\left(X_{p} \sim \bigcup_{z \in A_{n}} \bigcup_{i=1}^{l} E_{z i}^{n}\right)+\sum_{z \in A_{n}} \sum_{i=1}^{l n z}\left(1 / 2^{n}\right)|\mu|\left(E_{z i}^{n} \cap E\right) \\
& \leq \epsilon / 3
\end{array}
$$

and since $|\mu|\left(X_{p} \sim \bigcup_{z \in A_{n}} \bigcup_{i=1}^{l_{n z}} E_{z i}^{n}\right)<1 / 2^{n}$ we have by (ii) and (iii) that

$$
\left\|m(E)-\int_{E} f^{p} d \mu\right\| \leq \epsilon .
$$

Thus $m(E)=\int_{E} f^{p} d \mu$ for all $E \in \mathcal{S}_{p}$.

We now piece these functions together. Let $f: X \rightarrow B$ be defined by $f(t)=$ $\Sigma_{p=1}^{\infty} f^{p}(t)$ where we assume the functions are extended to be zero outside of $X_{p}$. Thus $X_{X_{p}} \cdot f=f^{p}$. It remains to be shown that $f \in \mathscr{L}_{B}(X, \mu)$ and that $m$ is the indefinite integral of $f$ with respect to $\mu$. 
Claim 5. $f \in \mathfrak{L}_{B}(X, \mu)$.

Proof. For each $p, 1 \leq p<\infty$, let $\left\{f_{n}^{p}\right\}_{n=1}^{\infty}$ be the sequence of stepfunctions which converge to $f^{p}$ on $X_{p}$ as defined earlier, extending them to be zero outside $X_{p}$. Define

$$
g_{n}(t)=\sum_{p=1}^{n} f_{n}^{p}(t)
$$

Now $\left\{g_{n}\right\}_{n=1}^{\infty}$ is a sequence of measurable stepfunctions which clearly converges to $f$ a.e. Thus it suffices to show that $\left\{g_{n}\right\}_{n=1}^{\infty}$ is Cauchy.

Let $\epsilon>0$ be given. Then there exists a $\delta>0$ such that $|\mu|(F)<\delta$ implies that $|m|(F)<\epsilon / 4$. Choose $N>0$ such that $|\mu|\left(X \sim \bigcup_{k=1}^{N} X_{k}\right)<\min \{\delta, \epsilon / 4\}$.

Now choose $M>N$ such that for all $m, n>M,\left\|f_{n}^{p}-f_{m}^{p}\right\|_{\rho} \leq \epsilon / 4 N$ for $1 \leq$ $p \leq N$

If $m>n \geq M$ we obtain

$$
\begin{aligned}
\left\|g_{n}-g_{m}\right\|_{\Phi} & =\sum_{p=1}^{N}\left\|f_{n}^{p}-f_{m}^{p}\right\|_{\perp}+\sum_{p=N+1}^{n}\left\|f_{n}^{p}-f_{m}^{p}\right\|_{\perp}+\sum_{p=n+1}^{m}\left\|f_{m}^{p}\right\|_{\perp} \\
& \leq \frac{\epsilon}{4}+\sum_{p=N+1}^{n}\left\|f_{n}^{p}\right\|_{\mathcal{L}}+\sum_{p=N+1}^{m}\left\|f_{m}^{p}\right\|_{\perp}
\end{aligned}
$$

and

$$
\left\|f_{k}^{p}\right\|_{\S} \leq\left(1 / 2^{n}\right)|\mu|\left(X_{p}\right)+|m|\left(X_{p}\right), \quad k=n, m,
$$

which implies

$$
\begin{aligned}
\left\|g_{n}-g_{m}\right\|_{\mathfrak{Q}} & \leq \frac{\epsilon}{4}+\left\{1 / 2^{n}+1 / 2^{m}\right\}|\mu|\left(X \sim \bigcup_{p=1}^{N} X_{p}\right)+2|m|\left(X \sim \bigcup_{p=1}^{N} X_{p}\right) \\
& \leq \frac{\epsilon}{4}+\frac{\epsilon}{4}+2 \frac{\epsilon}{4}=\epsilon .
\end{aligned}
$$

Thus $\left\{g_{n}\right\}_{n=1}^{\infty}$ is Cauchy and converges a.e., to $f$. Hence $f \in \mathscr{Q}_{B}(X, \mu)$.

Claim 6. If $E \in \mathcal{S}$, then $m(E)=\int_{E} f d \mu$.

Proof. Let $E \in \mathcal{S}$, then $E=\bigcup_{p=1}^{\infty} X_{p} \cap E, X_{p} \cap E \in \mathcal{S}_{p}$. Thus

$$
\int_{E} f d \mu=\sum_{p=1}^{\infty} \int_{X_{p} \cap E} f^{p} d \mu=\sum_{p=1}^{\infty} m\left(X_{p} \cap E\right)=m(E) .
$$

$(\Rightarrow)$. Suppose there exists $f \in \mathfrak{L}_{B}(X, \mu)$ such that $m(E)=\int_{E} f d \mu$ for all $E \in \mathcal{S}$. It is immediate that (i) and (ii) are valid, thus it remains only to demonstrate (iii).

Let $E \in \mathcal{S}^{+}$. Then by Lemma 1.5 there exists $F \subset E, F \in \mathcal{S}^{+}$, such that $\operatorname{er}_{F}(f)$ is relatively compact.

Claim 7. $F$ is localized in $K=\overline{\operatorname{er}_{F}(f)}$. 
Proof. Let $F^{\prime} \subset F, F^{\prime} \in \mathcal{S}^{+}$, and let $\epsilon>0$ be given. Choose $b \in \operatorname{er}_{F^{\prime}}(f)$ $\neq \varnothing\left[9\right.$, p. 469, Lemma 1.5] and let $\left.E^{\prime}=f^{-1}\left(S_{\epsilon}(b)\right) \cap F^{\prime} .{ }^{2}\right)$ Since $b \in \operatorname{er}_{F^{\prime}}(f)$, we have that $E^{\prime} \epsilon \mathcal{S}^{+}$. It thus suffices to show that $b \in A\left(E^{\prime}, \epsilon\right) \cap K$.

Clearly $b \in \operatorname{er}_{F}{ }^{o}(f) \subset K$, and $\|b-f(t)\|$ is $|\mu|$-integrable on $E^{\prime}$ since $\|b-f(t)\|$ is measurable and bounded. Thus if $F^{*} \subset E^{0}, F^{*} \in \mathfrak{S}^{+}$,

$$
\left\|\mu\left(F^{*}\right)(b)-m\left(F^{*}\right)\right\| \leq \int_{F^{*}}\|b-f(t)\| d|\mu|
$$

using Lemma 1.3 and since $f(t) \in S_{\epsilon}(b)$,

$$
\leq \int_{F^{*}} \epsilon d|\mu|=\epsilon|\mu|\left(F^{*}\right) \text {. }
$$

Thus $b \in A_{m}\left(E^{\prime}, \epsilon\right) \cap K$ and $F$ is localized in $K$.

The proof to this theorem yields two immediate results conceming $L_{B}^{1}(X, \mu)$ although the characterization of arbitrary indefinite integrals via this method appears so complicated as to be unmanagable for any conceivable application.

Corollary. Let $(X, \mathcal{S})$ be a measurable space and let $\mu: \mathcal{S} \rightarrow L\left[B, B_{1}\right]$ be a measure with total finite variation, where $B$ and $B_{1}$ are Banach spaces. Let $m: \mathfrak{S} \rightarrow B_{1}$ be a measure on $\mathcal{S}$. Then

(1) $m$ is the indefinite integral with respect to $\mu$ of $f \in L_{B}^{1}(X, \mu)$ such that $\operatorname{er}_{X}(f)$ is compact iff

(i) $m<<\mu$,

(ii) $|m|(X)<\infty$,

(iii) there exists a compact set $K \subset B$ such that $X$ is localized in $K$.

(2) $m$ is the indefinite integral with respect to $\mu$ of a bounded function $f \epsilon$ $L_{B}^{1}(X, \mu)$ iff

(i) $m \ll \mu$,

(ii) $|m|(X)<\infty$,

(iii) there exists a constant $M$ such that for eacb $E \in \mathcal{S}^{+}$there exists $F \subset E$, $F \in \mathcal{S}^{+}$, and a compact set $K \subset S_{M}(0) \subset B$ such that $F$ is localized in $K$.

3. Consequences. In this section we will derive Radon-Nikodým theorems for several special cases, some apparently unknown. The first setting is that of the Bochner integral and we shall obtain a theorem of Rieffel's [4, p. 466] and two different formulations.

Definition 3.1. If $A_{1}, \ldots, A_{n}$ are subsets in a Banach space $B$, then $\sigma\left(A_{1}, \ldots, A_{n}\right)=\left\{\sum_{i=1}^{n} \alpha_{i} x_{i} \mid \sum_{i=1}^{n} \alpha_{i}=1, \alpha_{i} \geq 0, x_{i} \in A_{i}, 1 \leq i \leq n\right\}$.

Definition 3.2. $A$ is called $\epsilon$-bounded if there exists $\left\{x_{1}, \ldots, x_{n}\right\} \subset B$ such that $A \subset \bigcup_{i=1}^{n} S_{\epsilon}\left(x_{i}\right)$.

Definition 3.3. If $E \in \mathcal{S}^{+}$, the average range of $m$ over $E$ is, $A_{E}(m)=$ $\left\{m(F) / \mu(F) \mid F \subset E, F \in S^{+}\right\}$.

The following technical lemma follows easily.

(2) $S_{\epsilon}(b)$ is the $\epsilon$ ball about $b$. 
Lemma 3.1. If $A_{n}, 1 \leq n \leq N$, are $\epsilon$-bounded then $\sigma\left(A_{1}, \ldots, A_{n}\right)$ is $2 \epsilon$-bounded.

The next theorem shows the equivalence of Rieffel's Radon-Nikodým theorem [4, p. 466], with two alternate theorems.

Theorem 3.1. Let $(X, \mathcal{S})$ be a measurable space and let $\mu$ be a totally finite positive measure on $\mathcal{S}$. Let $B$ be a Banach space and $m: \mathcal{S} \rightarrow B$ a $\mu$-continuous $B$-valued measure with totally finite variation. Then the following three conditions are equivalent:

(i) given $E \in \mathcal{S}^{+}$, there is an $F \subset E, F \in \mathcal{S}^{+}$, such that $A_{F}(m)$ is relatively compact,

(ii) given $E \in \mathcal{S}^{+}$and $\epsilon>0$ there is an $F \subset E, F \in \mathcal{S}^{+}$, sucb that $A_{m}(F, \epsilon)$ $\neq \varnothing$, and

(iii) given $E \in \mathcal{S}^{+}$and $\epsilon>0$ there is an $F \subset E, F \in \mathcal{S}^{+}$, such that the diameter of $A_{F}(m) \leq \epsilon$.

Proof. (i) $\Rightarrow$ (ii). This follows from an argument of Rieffel's [4, p. 475] showing that $\exists b$ which is $(F, \epsilon / 2)$ opure, in other words that $m\left(F^{*}\right) / \mu\left(F^{*}\right) \epsilon$ $S_{\epsilon}(b)$ for all $F^{*} \in \mathcal{S}^{+}, F^{*} \subset F$, which implies $\left|m\left(F^{*}\right) \ldots b \mu\left(F^{*}\right)\right|<\epsilon \mu\left(F^{*}\right)$.

(ii) $\Rightarrow$ (iii). This is immediate since $b \in A_{m}(F, \epsilon / 2)$ implies that $\left\|m\left(F^{*}\right) / \mu\left(F^{*}\right)-b\right\|<\epsilon / 2$ for all $F^{*} \subset F, F^{*} \in \mathcal{S}^{+}$.

(iii) $\Rightarrow$ (i). Let $E \in \mathcal{S}^{+}$be given. Now condition (iii) implies that the diameter $\left(A_{F}(m)\right) \leq \epsilon$ is a local null difference property. Hence given any $G \in \mathcal{S}^{+}$ and any $\epsilon>0$ we can decompose $G$ by Lemma 1.1 in the following manner:

$G=\bigcup_{i \in I} G_{i}, G_{i} \in \mathcal{S}^{+}$, diameter $\left(A_{G_{i}}(m)\right) \leq \epsilon, i \in I$. Let $a=\mu(E)$. Then by above there exists a disjoint collection of subsets of $E,\left\{F_{i}^{1}\right\}_{i=1}^{N} \subset \mathcal{S}^{+}$such that diameter $\left(A_{F_{i}^{1}}(m)\right) \leq 1 / 2$ and $\mu\left(E \sim \bigcup_{i=1}^{N_{1}} F_{i}^{1}\right)<\alpha / 4$. Let $F_{1}=\bigcup_{i=1}^{N} F_{i}^{1}$.

We continue by induction. If at the $n$th step $F_{n} \in \mathcal{S}^{+}, \mu\left(F_{n}\right)>$ $\left[\left(2^{n}+1\right) / 2^{n+1}\right] \alpha$, then there exists a disjoint collection of subsets of $E$, $\left\{F_{i}^{n+1}\right\}_{i=1}^{N} \subset+1$ $\left(F_{i}^{n+1}\right)<1 / 2^{n+1}$. Let $F_{n+1}=\bigcup_{i=1}^{N_{n+1}} F_{i}^{n+1}$ and we have that

$$
\mu\left(F_{n+1}\right)>\frac{2^{n}+1}{2^{n+1}} \alpha-\frac{a}{2^{n+2}}=\frac{2^{n+1}+1}{2^{n+2}} a .
$$

Therefore we have constructed a sequence $F_{1} \supset F_{2} \supset \cdots$ such that $\mu\left(F_{n}\right)>$ $\left[\left(2^{n}+1\right) / 2^{n+1}\right] a$, and such that $A_{F_{n}}(m)$ is $1 / 2^{n-1}$-bounded. Let $F=\bigcap_{n=1}^{\infty} F_{n^{\prime}}$, then $F \in \mathcal{S}^{+}$since $\mu(F) \geq a / 2$.

Claim 1. $A_{F}(m) \subset \bigcap_{n=1}^{\infty} \sigma\left(A_{F_{1}^{n}}(m), \ldots, A_{F_{N}^{n}}(m)\right)$.

Proof. Let $F_{0} \subset F, F_{0} \in \mathcal{S}^{+}$. Then $F_{0} \subset \bigcup_{i=1}^{N_{n}} F_{i}^{n}$ and 


$$
\frac{m\left(F_{0}\right)}{\mu\left(F_{0}\right)}=\sum_{i=1}^{N_{n}} \frac{\mu\left(F_{0} \cap F_{i}^{n}\right)}{\mu\left(F_{0}\right)}\left[\frac{m\left(F_{0} \cap F_{i}^{n}\right)}{\mu\left(F_{0} \cap F_{i}^{n}\right)}\right]
$$

where we use the convention $0 / 0=0$. Now

$$
\sum_{i=1}^{N} \frac{\mu\left(F_{0} \cap F_{i}^{n}\right)}{\mu\left(F_{0}\right)}=1 \quad \text { and } \quad \frac{m\left(F_{0} \cap F_{i}^{n}\right)}{\mu\left(F_{0} \cap F_{i}^{n}\right)} \in A_{F_{i}^{n}}^{(m)}
$$

when $\mu\left(F_{0} \cap F_{i}^{n}\right) \neq 0$. If $\mu\left(F_{0} \cap F_{i}^{n}\right)=0$ then we can replace $m\left(F_{0} \cap F_{i}^{n}\right) / \mu\left(F_{0} \cap F_{i}^{n}\right)$ by any element of $A_{F_{i}^{n}}(m)$. Thus for all $n$,

$$
\frac{m\left(F_{0}\right)}{\mu\left(F_{0}\right)} \in \sigma\left(A_{F_{1}^{n}}^{(m)}, \cdots, A_{F_{N}^{n}}^{n}(m)\right) .
$$

Claim 2. $A_{F}(m)$ is relatively compact.

Proof. For every $n, A_{F_{i}^{n}}(m)$ is $1 / 2^{n}$ bounded and hence by Lemma 3.1, $\sigma\left(A_{F_{1}^{n}}(m), \ldots, A_{F_{N_{n}}^{n}}(m)\right)$ is $1 / 2^{n-1}$ bounded which implies that $A_{F}(m)$ is $1 / 2^{n+1}$-bounded. Thus $A_{F}(m)$ is totally bounded and hence relatively compact.

Corollary (Rieffel [4, p. 466]). Let $(X, \delta)$ be a measurable space and let $\mu$ be a totally finite positive measure on $\mathcal{S}$. If $m$ is a $B$-valued measure on $\mathcal{S}, B$ a Banach space, then $m$ is the indefinite integral with respect to $\mu$ of a B-valued Bochner integrable function on $X$ iff

(i) $m<<\mu$,

(ii) $|m|(X)<\infty$,

(iii) for each $E \in \mathfrak{S}^{+}$, there is an $F \subset E, F \in \mathfrak{S}^{+}$, sucb that $A_{F}(m)$ is relatively compact.

Remark. Theorem 3.1 gives two equivalent conditions to condition (iii) and in fact the locally small average range condition, condition (iii) of Theorem 3.1, seems to be the most useful of the conditions. This condition is the closest condition to the dentable condition of Rieffel [5, p. 71].

Proof of corollary. By Theorem 1.1, $\mathcal{\complement}_{B}(X, \mu)=L_{B}^{1}(X, \mu)$, and hence it suffices to show the equivalence between (iii) and condition (iii) of Theorem 2.1. The necessity of (iii) follows immediately from Theorem 3.1.

Suppose $m$ satisfies (i), (ii), and (iii), and $E \in \mathcal{S}^{+}$. Choose $F \subset E$ such that $A_{F}(m)$ is relatively compact and let $K_{F}=\overline{A_{F}(m)}$, which is compact.

Claim. $F$ is localized in $K_{F}$.

Proof. Let $F^{\prime} \subset F, F^{\prime} \in \mathcal{S}^{+}$. Then by Theorem 3.1 there exists $E^{\prime} \subset F^{\prime}$, $E^{\prime} \in \mathcal{S}^{+}$, such that the diameter $\left(A_{E^{\prime}}(m)\right)<\epsilon$. Thus $A_{E^{\prime}}(m) \subset A\left(E^{\prime}, \epsilon\right)$ and since $A_{E^{\prime}}(m) \subset A_{F}(m) \subset K_{F}$ we have $A\left(E^{\prime}, \epsilon\right) \cap K_{F} \neq \varnothing$. Thus $F$ is localized in $K_{F^{*}}$.

We will now consider a different setting; one in which, as far as we know, no 
Radon-Nikodým theorems of this type were previously known.

Let $\mu$ be a $B$ valued measure where $B$ is again a Banach space. Then by identifying $B$ with $L[C, B]$ in the natural manner, we can use our general theorem to characterize the indefinite integrals of scalar functions with respect to $\mu$. As in the case of the Bochner integral setting, $L^{1}(X, \mu)=\mathscr{L}_{C}(X, \mu)$ which was shown in Theorem 1.1.

After obtaining this characterization we will then demonstrate the simplification which results when $\mu$ is a measure defined by density with a positive base.

We note the following trivial fact concerning variation before stating the main result.

Lemma 3.2. If $E \in \mathfrak{S}^{+}$, then for any $\epsilon>0$ there exists $F \subset E, F \in \mathcal{S}^{+}$, such that $\|\mu(F)\| \neq 0$ and $|\mu|(F) /\|\mu(F)\|<1+\epsilon$.

Theorem 3.2. Let $(X, \mathcal{S})$ be a measurable space and $\mu: \mathcal{S} \rightarrow B$, where $B$ is a Banach space, be a measure with totally finite variation. Let $m$ be a B-valued measure on $\mathcal{S}$. Then $m$ is the indefinite integral with respect to $\mu$ of a C.valued integrable function on $X$ iff

(i) $m<<\mu$,

(ii) $|m|(X)<\infty$,

(iii) for all $E \in \mathcal{S}^{+}$, and for all $\epsilon>0$, there exists $F \subset E, F \in \mathfrak{S}^{+}$, such that $A(F, \epsilon) \neq \varnothing$.

Proof. Again it suffices to show the equivalence between condition (iii) and condition (iii) of Theorem 2.1. The necessity is immediate.

Let $E \in \mathfrak{S}^{+}$and by (iii) there exists $F \subset E, F \in \mathfrak{S}^{+}$, such that $A(F, 1) \neq \varnothing$. Pick $x \in A(F, 1)$ and let $K_{F}=\overline{S_{2}(x)}$, the closed ball about $x$ with radius 2 , which is compact since $K_{F} \subset \mathrm{C}$.

Claim. $F$ is localized in $K_{F}$.

Proof. Let $F_{0} \subset F, F_{0} \in \mathcal{S}^{+}$. We need to show that $F_{0}$ is semilocalized in $K_{F}$. Let $\epsilon>0$ be given. Then by (iii) there exists $F^{\prime} \subset F_{0}, F^{\prime} \in \mathcal{S}^{+}$, such that $A\left(F^{\prime}, \epsilon\right) \neq \varnothing$. Let $y \in A\left(F^{\prime}, \epsilon\right)$, we will show that $y \in \overline{S_{2}(x)}=K_{F}$. If $\epsilon \geq 1$ we have that $A(F, 1) \subset A\left(F^{\prime}, \epsilon\right)$ and hence that $A\left(F^{\prime}, \epsilon\right) \cap K_{F} \neq \varnothing$.

If $\epsilon<1$, then for any $\gamma>0$, we can find $E_{0} \subset F^{\prime}, E_{0} \in \mathfrak{S}^{+}$, such that $|\mu|\left(E_{0}\right) /\left\|\mu\left(E_{0}\right)\right\|<1+\gamma$ by Lemma 3.2. Hence,

$$
|x-y| \leq \frac{|\mu|\left(E_{0}\right)}{\left\|\mu\left(E_{0}\right)\right\|}\left\{\left\|x \frac{\mu\left(E_{0}\right)}{|\mu|\left(E_{0}\right)}-\frac{m\left(E_{0}\right)}{|\mu|\left(E_{0}\right)}\right\|+\left\|\frac{m\left(E_{0}\right)}{|\mu|\left(E_{0}\right)}-y \frac{\mu\left(E_{0}\right)}{|\mu|\left(E_{0}\right)}\right\|\right\}
$$

and since $x \in A(F, 1)$ and $y \in A\left(F^{\prime}, \epsilon\right)$, this yields

$$
|x-y| \leq(1+\gamma)\{1+\epsilon\} \leq 2(1+\gamma) \text {. }
$$


Since $\gamma$ is arbitrary, $|x-y| \leq 2$. Thus $y \in \overline{S_{2}(x)}=K_{F}$ and $A\left(F^{\prime}, \epsilon\right) \cap K_{F} \neq \varnothing$.

Therefore we have shown that $F_{0}$ is semilocalized in $K_{F}$ and hence that $F$ is localized in $K_{F}$.

In the more general case where $\mu$ is $L\left[B, B_{1}\right]$-valued but $B$ is only finite dimensional we get, using similar arguments, the same result. It is important to notice that, in this instance, $\mathfrak{Q}_{B}(X, \mu) \neq L_{B}^{1}(X, \mu)$ in general.

Theorem 3.3. Let $(X, \mathcal{S})$ be a measurable space and $\mu: \mathcal{S} \rightarrow L\left[B, B_{1}\right]$, where $B, B_{1}$ are Banacb spaces and $B$ is finite dimensional, be a measure on $\mathcal{S}$ with totally finite variation. Let $m$ be a $B_{1}$-valued measure on $\mathcal{S}_{\text {. Then }} m$ is the indefinite integral with respect to $\mu$ of a function $f \in \mathfrak{L}_{B}(X, \mu)$ iff

(i) $m<<\mu$

(ii) $|m|(X)<\infty$,

(iii) for all $E \in \mathfrak{S}^{+}$and for all $\epsilon>0$, there exists $F \subset E, F \in \mathcal{S}^{+}$, sucb that $A(F, \epsilon) \neq \varnothing$.

Returning to the case where $B=C$, if $\mu$ happens to be a measure defined by density [1, p. 165] with positive base; i.e., an indefinite Bochner integral, then much simpler results can be obtained.

Definition 3.4. If $E \epsilon \delta^{+}$and $\epsilon>0$ we define

$$
B(E, \epsilon)=\left\{r \in \mathbf{C}\left|\quad F \subset E, F \in S^{+},\|\mu(F)\| \neq 0 \ni\|m(F)-r \mu(F)\| \leq \epsilon\right| \mu \mid(F)\right\} .
$$

The following lemma is merely a consolidation of Theorem 3.1 and its corollary.

Lemma 3.3. If $\mu(E)=\int_{E} f d \nu$, where $\nu$ is totally finite positive measure, $f \in L_{B}^{1}(X, \nu)$ and $B$ is a Banach space, then for all $\epsilon>0$ and $E \epsilon \mathcal{S}^{+}$, there exists $F \subset E, F \in \mathcal{S}^{+}$, such that $A_{F}(\mu)$ is relatively compact and the diameter $\left(A_{F}(\mu)\right)$ $\leq \epsilon$.

In the following material we will let $A_{F}^{\nu}(\mu)$ denote the average range of $\mu$ with respect to $\nu$.

Lemma 3.4. If $\mu$ is as in Lemma 3.3, then for each $E \in \mathcal{S}^{+}$, there exists $a>$ 0 and $F \subset E, F \in \mathcal{S}^{+}$, such that $A_{F}^{\nu}(\mu) \cap S_{\alpha}(0)=\varnothing$.

Proof. Choose $E^{\prime} \subset E, E^{\prime} \ni \mathcal{S}^{+}$, such that $\left\|\mu\left(E^{\prime}\right)\right\|>0$ and since $\nu\left(E^{\prime}\right)>0$ we can let $\gamma=\left\|\mu\left(E^{\prime}\right)\right\| / \nu\left(E^{\prime}\right)>0$.

Claim. There exists $F \subset E^{\prime}, F \in \mathfrak{S}^{+}$, such that

$$
A_{F}^{\nu}(\mu) \cap S_{\gamma / 2}(0)=\varnothing \text {. }
$$

Proof. Suppose not. Then the property that $\|\mu(F)\| / \nu(F) \leq \gamma / 2$ and diameter diameter $\left(A_{F}^{\nu}(\mu)\right)<\gamma_{\prime}^{\prime} 2$ is a local, null difference property. Thus by Lemma $1.1, E^{\prime}=$ $\bigcup_{i \in I} E_{i},\left\{E_{i}\right\}_{i \in I} \subset \mathcal{S}^{+}$, disjoint, $\left\|\mu\left(E_{i}\right)\right\| / \nu\left(E_{i}\right) \leq \gamma / 2$, and diameter $\left(A_{E_{i}}^{\nu}(\mu)\right)<$ $\gamma / 4$. 
But this implies that

$$
\bigcup_{i=1}^{\infty} A_{E_{i}}^{\nu}(\mu) \subset S_{3 \gamma / 4}(0)
$$

Hence $\bar{c}\left(\bigcup_{i=1}^{\infty} A_{E_{i}}^{\nu}(\mu)\right) \subset \overline{S_{3 \gamma / 4}(0)}$, but

$$
\frac{\mu\left(E^{\prime}\right)}{\nu\left(E^{\prime}\right)}=\sum_{i=1}^{\infty} \frac{\nu\left(E_{i}\right) \mu\left(E_{i}\right)}{\nu\left(E^{\prime}\right)} \frac{\bar{\nu}\left(E_{i}\right)}{\bar{c}}\left(\bigcup_{i=1}^{\infty} A_{E_{i}}^{\nu}(\mu)\right),
$$

where $\vec{c}(A)$ represents the closed convex hull of $A$. Thus $\left\|\mu\left(E^{\prime}\right)\right\| / \nu\left(E^{\prime}\right) \leq 3 \gamma / 4$ which is a contradiction.

The following theorem actually characterizes indefinite integrals of measures which are themselves indefinite Bochner integrals, since if $\mu$ is an indefinite Bochner integral then it is an indefinite Bochner integral with respect to its own variation.

Theorem 3.4. Let $(X, \mathfrak{S})$ be a measurable space and let $\mu: \mathfrak{S} \rightarrow B$ be a measure on $\mathfrak{S}$ with finite variation. Suppose further that there exists a $g \in$ $L_{B}^{1}(X, \mu)$ such that $\mu(E)=\int_{E} g d|\mu|$, for all $E \in \mathcal{S}$. Let $m$ be a B-valued measure on $\mathcal{S}$, then $m$ is the indefinite integral with respect to $\mu$ of a $\mathrm{C}$-valued integrable function on $X$ iff

(i) $m<<\mu$,

(ii) $|m|(X)<\infty$,

(iii) for eacb $E \in \mathcal{S}^{+}$and $\epsilon>0, B(E, \epsilon) \neq \varnothing$, and

(iv) for each $E \in \mathfrak{S}^{+}$, there exists $F \subset E, F \in \mathfrak{S}^{+}$, such that $\left.A_{F}\right|^{\mu \mid}(m)$ is relatively compact.

Remark. Any of the equivalent conditions in Theorem 3.1 could be substituted for condition (iv).

Proof. It suffices to show, under these hypotheses, that condition (iii) and (iv) are equivalent to condition (iii) of Theorem 3.2.

The necessity follows immediately from the fact that $A(F, \epsilon) \subset B(F, \epsilon)$ and that if $\mu(E)=\int_{E} g d|\mu|$ and $m(E)=\int_{E} f d_{\mu}$ then $m(E)=\int_{E} f g d|\mu|$ by $[1$, p. 170, Corollary 1]. There is also a short self-contained proof of the necessity.

In order to prove the sufficiency we need to show that for $E \in \mathcal{S}^{+}$and $\epsilon>0$ that there exists $F \subset E, F \in \mathcal{S}^{+}$, such that $A(F, \epsilon) \neq \varnothing$.

Claim 1. There exists $F \subset E, F \in \mathcal{S}^{+}$, and a $\delta>0$ such that the diameter $(B(F, \delta))<\epsilon / 2$.

Proof. Now by the corollary to Theorem 2.1 there exists $E_{1} \subset E, E_{1} \in \mathcal{S}^{+}$, such that $A_{E_{1}}^{|\mu|}(\mu)$, and $A_{E_{1}}^{\mid \mu}(m)$ are relatively compact. Thus there exists $R>0$ which is a norm bound for both sets. Now by Lemma 3.4 there exists $r, 0<r<1$, and $F^{\prime} \subset E_{1}, F^{\prime} \in \mathcal{S}^{+}$, such that $A_{F}^{\prime}|\mu|(\mu) \cap S_{r}(0)=\varnothing$.

We now obtain a bound on the elements of $B\left(F^{\prime}, \epsilon\right)$. If $x \in B\left(F^{\prime}, \epsilon\right)$ then there 
exists $\widetilde{E} \subset F^{\prime}, \widetilde{E} \in \mathcal{S}^{+},\|\mu(\breve{E})\| \neq 0$, such that $\|m(\widetilde{E})-x \mu(\widetilde{E})\| \leq \epsilon|\mu|(\widetilde{E})$.

$$
|x| \leq \epsilon \frac{|\mu|(\widetilde{E})}{\|\mu(\widetilde{E})\|}+\frac{|\mu|(\widetilde{E})}{\|\mu(\widetilde{E})\|}\left\|\frac{m(\widetilde{E})}{|\mu|(\widetilde{E})}\right\| \leq \epsilon / r+R / r=[R+\epsilon] / r=N .
$$

Applying condition (iv) and Theorem 2.1 and its corollary, there exists $F \subset F^{\prime}, F \in \mathcal{S}^{+}$, such that

(i) diameter $\left(A_{F}^{|\mu|}(m)\right)<\epsilon r / 8$,

(ii) diameter $(A|\mu|(\mu))<\epsilon r / 8 N$.

We now show that $\delta=\epsilon r / 8$ and $F$ satisfy the claim.

Choose $x, y \in B(F, \epsilon r / 8)$. Then there exists $\widetilde{F} \subset F,\|\mu(\widetilde{F})\|>0, \widetilde{E} \subset F$, $\|\mu(\widetilde{E})\|>0$, such that

$$
\|m(\widetilde{F})-x \mu(\widetilde{F})\| \leq \frac{\epsilon r}{8}|\mu|(\widetilde{F}) \text { and }\|m(\widetilde{E})-y \mu(\widetilde{E})\| \leq \frac{\epsilon r}{8}|\mu|(\widetilde{E}) .
$$

Thus using the previously established bounds,

$$
\begin{aligned}
|x-y|= & \frac{|\mu|(\widetilde{F})}{\|\mu(\widetilde{F})\|}\left[\left\|x \frac{\mu(\widetilde{F})}{|\mu|(\widetilde{F})}-\frac{m(\widetilde{F})}{|\mu|(\widetilde{F})}\right\|+\left\|\frac{m(\widetilde{F})}{|\mu|(\widetilde{F})}-\frac{m(\widetilde{E})}{|\mu|(\widetilde{E})}\right\|\right. \\
& \left.+\left\|\frac{m(\widetilde{E})}{|\mu|(\widetilde{E})}-y \frac{\mu(\widetilde{E})}{|\mu|(\widetilde{E})}\right\|+|y|\left\|\frac{\mu(\widetilde{E})}{|\mu|(\widetilde{E})}-\frac{\mu(\widetilde{F})}{|\mu|(\widetilde{F})}\right\|\right] \\
< & <[\epsilon r / 8+\epsilon r / 8+\epsilon r / 8+N \cdot \epsilon r / 8 N] / r=\epsilon / 2 .
\end{aligned}
$$

Thus the diameter $(B(F, \delta))<\epsilon / 2$.

Claim 2. $B(F, \delta) \subset A(F, \epsilon)$.

Proof. Let $x \in B(F, \delta)$ and $F^{\prime} \subset F, F^{\prime} \in \mathcal{S}^{+}$, then we need to show that $\left\|m\left(F^{\prime}\right)-x \mu\left(F^{\prime}\right)\right\|<\epsilon|\mu|\left(F^{\prime}\right)$. Consider the set property $P: E \in \mathcal{S}^{+}$has property $P$ if there exists $r \in B(F, \delta)$ such that $\|m(E)-r \mu(E)\|<\delta|\mu|(E)$. P is a local property in $F^{\prime}$ since if $\widetilde{F} \subset F^{\prime}, \widetilde{F} \in \mathcal{S}^{+}$, we have by (iii) that $B(\widetilde{F}, \delta) \neq \varnothing$ and since $B(\widetilde{F}, \delta) \subset B\left(F^{\prime}, \delta\right)$, there exists $F^{*} \subset \widetilde{F},\left\|\mu\left(F^{*}\right)\right\|>0$, and $r \in B\left(F^{\prime}, \delta\right)$ such that $\left\|m\left(F^{*}\right)-r \mu\left(F^{*}\right)\right\|<\delta|\mu|\left(F^{*}\right), P$ is clearly a null difference property.

Thus by Lemma 1.1, $F^{\prime}=\bigcup_{i \in I} E_{i},\left\{E_{i}\right\}$ disjoint, such that there exists $r_{i}, i \in I$, such that $\left\|m\left(E_{i}\right)-r_{i} \mu\left(E_{i}\right)\right\| \leq \delta$.

Let $\gamma>0$ be arbitrary. Then there exists $M$ such that $|\mu|\left(F^{\prime} \sim \bigcup_{i=1}^{M} E_{i}\right)$ $<\gamma / 2 N$ and such that $|m|\left(F^{\prime} \sim \bigcup_{i-1}^{M} E_{i}\right)<\gamma / 2$. Then

$$
\begin{aligned}
\left\|m\left(F^{\prime}\right)-x \mu\left(F^{\prime}\right)\right\| & \leq|m|\left(F^{\prime} \sim \bigcup_{i=1}^{M} E_{i}\right)+|x| \cdot|\mu|\left(F^{\prime} \sim \bigcup_{i=1}^{M} E_{i}\right)+\sum_{i=1}^{M}\left\|m\left(E_{i}\right)-x \mu\left(E_{i}\right)\right\| \\
& \leq \frac{\gamma}{2}+N \frac{\gamma}{2} \bar{N}+\sum_{i=1}^{M}\left\|m\left(E_{i}\right)-r_{i} \mu\left(E_{i}\right)\right\|+\sum_{i=1}^{M}\left|x-r_{i}^{\prime}\right| \mu \mid\left(E_{i}\right),
\end{aligned}
$$

and since the diameter $(B(F, \delta))<\epsilon / 2$, 


$$
\leq \gamma+\sum_{i=1}^{M} \delta|\mu|\left(E_{i}\right)+\frac{\epsilon}{2} \sum_{i=1}^{M}|\mu|\left(E_{i}\right) .
$$

Thus since $\delta=\epsilon r / 8<\epsilon / 8$,

$$
\left\|m\left(F^{\prime}\right)-x \mu\left(F^{\prime}\right)\right\| \leq \gamma+\epsilon|\mu|\left(\bigcup_{i=1}^{M} E_{i}\right)<\gamma+\epsilon|\mu|\left(F^{\prime}\right) .
$$

Since $\gamma>0$ is arbitrary, we have that $\left\|m\left(F^{\prime}\right)-x \mu\left(F^{\prime}\right)\right\| \leq \epsilon|\mu|\left(F^{\prime}\right)$. Thus $x \in A(F, \epsilon)$.

We have thus established the result.

\section{BIBLIOGRAPHY}

1. N. Dinculeanu, Vector measures, Internat. Series of Monographs in Pure and Appl. Math., vol. 95, Pergamon Press, Oxford; VEB Deutscher Verlag der Wissenschaften, Berlin, 1967. MR $34 \# 6011 \mathrm{~b}$.

2. N. Dunford and J. T. Schwartz, Linear operators. I: General theory, Pure and Appl. Math., vol. 7, Interscience, New York, 1958. MR 22 \#302.

3. M. Métivier, Martingales à valeurs vectorielles. Applications à la dérivation des mesures vectorielles, Ann. Inst. Fourier (Grenoble) 17 (1967), fasc. 2, 175-208. MR 40 \#926.

4. M. A. Rieffel, The Radon-Nikody'm theorem for the Bochner integral, Trans. Amer. Math. Soc. 131 (1968), 466-487. MR 36 \#5297.

5. - Dentable subsets of Banach spaces, with application to a Radon-Nikodým theorem, Proc. Conference Functional Analysis (Irvine, Calif., 1966), Academic Press, London; Thompson, Washington, D. C., 1967, pp. 71-77. MR 36 \#5668.

6. J. J. Uhl, Jr., Applications of Radon-Nikodým theorems to martingale convergence, Trans. Amer. Math. Soc. 145 (1969), 27 1-285. MR 40 \#4983. 